\title{
nature
}

photonics

\section{Natural light}

\section{Nature has had millions of years to perfect the art of playing with light. Her intricate designs are providing useful fodder for our imagination.}

Mention the words 'optical technology' and images of LEDs, lasers and optical fibres come to mind - all man-made devices. But artificial is not always best: when developing new technology it should be remembered that evolution has had millions of years to perfect biological structures that interact with light. Increasingly, it turns out that nature relies on some rather complex, elegant structures - often with a simple yet ingenious design — to manipulate light in useful ways.

Take butterflies for instance. Scientists are looking to the delicate wings of this creature for inspiration for their latest highperformance optical devices. Why? Some Morpho butterflies, for example, signal to each other by giving off a dazzling blue-green hue that is visible up to half a mile away. They achieve this by reflecting light in just the right way, through the use of nanostructures embedded within their wing scales.

On page 123 of this issue ${ }^{1}$, researchers from General Electric Global Research Centre show there is more to butterflies' optical powers than just pretty colours. The way in which their wing structures reflect light is determined by the outside environment - and it has now been shown that the wing scales of certain Morpho species give a different optical response depending on the type of vapour present. As one of the authors tells us ${ }^{2}$, by mimicking these structures in man-made devices we might be able to replace complicated sensor arrays with highly selective, simple chemical sensors that can sniff out gases with unprecedented performance.

Butterflies are not the only ones to play with light. Their cousins, the moths, have also evolved structures that use light in innovative ways, as have fish, birds, plants and even some algae ${ }^{3}$. Rightly so, the focus on photonic structures found in nature is diversifying

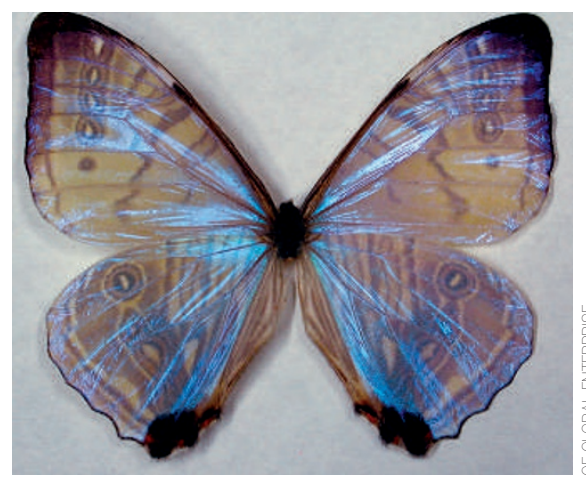

level, with the potential for intense light beams confined on a previously inaccessible tiny scale. The fibres feature a tiny hole just a few hundred nanometres in diameter located in the centre of an optical fibre's solid glass core - an impressive feat of fabrication. As for the applications of such fibres, it is an opportunity for researchers to let their imagination run wild. From our profile of Teruo Hiruma ${ }^{4}$, the president of Hamamatsu Photonics, it is clear that imagination has played a big role in the leaps and bounds he has made over the past 50 years.

Elsewhere in our second serving of and has piqued the curiosity of materials scientists, engineers and physicists in both academic institutions and industry. Those pushing the boundaries of technology would do well to step outside the lab and look at the world around them for inspiration.

Engineering - whether it be from nature herself or by the human hand - often involves design trade-offs: one component of a device has to make room for another. A breakthrough in one particular element can revolutionize the operation of the overall device, and this is illustrated nicely on page 119 of this issue, where scientists from the University of California, Berkeley demonstrate a new type of mirror that could turn heads in the laser world. They replace the conventional reflector inside a laser cavity with a tiny grating structure that offers a broadband mirror in a more compact package. Ultimately, this hi-tech reflector could make lasers a lot smaller, less power-hungry and lead to their inclusion in an even wider range of optical devices.

The careful design and fabrication prowess demonstrated above are also common themes in another of this month's papers. On page 115 a team from England and Brazil report a new type of optical fibre that can guide light at a subwavelength
Nature Photonics, we focus on another fertile ground for technology: terahertz optics ${ }^{5,6}$. Terahertz (THz) pioneers are exploring in earnest the 'THz gap' - a previously forgotten region of the electromagnetic spectrum that spans wavelengths from the infrared to microwaves. Although initially driven by applications in astronomy and analytical science, $\mathrm{THz}$ physics has grown to become a hot topic for the medical, security and pharmaceutical industries who see market opportunities by the dozen. These socalled T-rays can pass through objects easily, detect drugs and explosives, identify cancers and even watch paint dry (something that car makers actually have an avid interest in). As one researcher puts it?: "Terahertz research is one of the most intriguing and challenging fields to emerge in the 21 st century." Have a read and see for yourself.

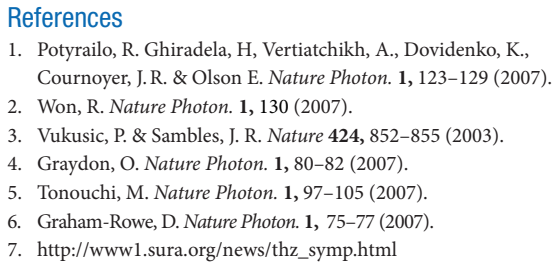

